\title{
Conhecimento da Equipe de Enfermagem sobre o Processo de hemotransfusão:
}

\section{Revisão Integrativa da Literatura}

\section{Knowledge of the Nursing Team on the Blood Transfusion Process: Integrative Literature Review \\ Conocimiento del equipo de enfermería sobre el proceso de transfusión sanguínea: Revisión}

integradora de la literatura

Ernestina Nazaré Cardoso Alves ORCID: https://orcid.org/0000-0002-9334-0148 Universidade do Estado do Pará, Brasil E-mail: encapaty00@gmail.com

Marcela Raíssa Asevedo Dergan ORCID: https://orcid.org/0000-0003-1457-0242 Universidade do Estado do Pará, Brasil E-mail: derganm20@gmail.com

Diélig Teixeira

ORCID: https://orcid.org/0000-0002-0520-6759 Hospital Adventista de Belém, Brasil E-mail: dielig.enf@outlook.com

Francinéa de Nazaré Ferreira de Castilho ORCID: https://orcid.org/0000-0002-9278-0010

Universidade do Estado do Pará, Brasil

E-mail: francineacastilho@hotmail.com Iranete Pereira Ribeiro

ORCID: https://orcid.org/0000-0002-0135-2206 Hospital Ophyr Loiola, Brasil E-mail: iraneterib@gmail.com Nádia Oliveira Aires ORCID https://orcid.org/0000-0001-9579-0110 Centro Universitário Metropolitano da Amazônia, Brasil E-mail: nadyya_aires@hotmail.com

Yves de Luka Miranda dos Santos ORCID: https://orcid.org/0000-0002-1244-6056 Centro Universitário Metropolitano da Amazônia, Brasil E-mail: lukamiranda0@gmail.com

Elineide Ferreira Brasil do Nascimento ORCID: https://orcid.org/0000-0003-2944-6877 Universidade da Amazônia, Brasil E-mail: neydebrasil@hotmail.com

Beatriz Borges Araújo

ORCID: https://orcid.org/0000-0001-6052-9262 Universidade da Amazônia, Brasil E-mail: bia.borges2169@gmail.com

Lucas de Jesus Pereira ORCID: https://orcid.org/0000-0002-3938-3286 Universidade de São Paulo, Brasil E-mail: lucas-laje@hotmail.com

Larissa Graziela Sousa da Silva ORCID: https://orcid.org/0000-0002-5853-334X Hospital Universitário Getúlio Vargas, Brasil

E-mail: laragazi@gmail.com

Odilene Silva Costa

ORCID: https://orcid.org/0000-0002-7714-5078 Instituto de assistência à saúde dos servidores públicos do município de Belém, Brasil

E-mail: odilenesilva@ hotmail.com

Thalita Mariana Gonçalves da Silva ORCID: https://orcid.org/0000-0003-1504-1596 Hospital Ophir Loyola, Brasil

E-mail: Thatanery@ hotmail.com

Ingrid Melo de Menezes

ORCID: https://orcid.org/0000-0001-9249-9141 Hospital Pró Infantil, Brasil

E-mail: ingridmelooenfr@gmail.com 
Iara do Nascimento Pantoja

ORCID: https://orcid.org/0000-0003-2889-3552 Universidade Federal do Pará, Brasil E-mail: iarapantoja12@hotmail.com

Dayara de Nazaré Rosa de Carvalho ORCID: https://orcid.org/0000-0001-8569-3392 Universidade Estadual do Pará, Brasil E-mail: dayara_twain@hotmail.com Maicon de Araújo Nogueira

ORCID: https://orcid.org/0000-0002-8315-4675 Universidade do Estado do Pará, Brasil

E-mail: profmaiconnogueira@gmail.com Antônia Margareth Moita Sá

ORCID: https://orcid.org/0000-0002-2053-5622 Universidade do Estado do Pará, Brasil E-mail: margarethsa@gmail.com

Maria Elizabete de Castro Rassy ORCID: https://orcid.org/0000-0002-7214-5079 Fundação Santa Casa de Misericórdia do Pará, Brasil E-mail: elizarassy50@hotmail.com

Heliana Helena de Moura Nunes

ORCID: https://orcid.org/0000-0003-2263-5755 Fundação Santa Casa de Misericórdia do Pará, Brasil E-mail: heliana_moura@ hotmail.com

\begin{abstract}
Resumo
Objetivo: Identificar os estudos científicos sobre o conhecimento da equipe de enfermagem diante de uma transfusão sanguínea; observar qual o grau de conhecimento da equipe de enfermagem referente à hemotransfusão; identificar as ferramentas de gerenciamento de risco que possam contribuir para esse conhecimento. Metodologia: Trata-se de uma Revisão Integrativa de Literatura, exploratória com abordagem qualitativa sobre o conhecimento da equipe de enfermagem sobre hemotransfusão, realizada nas bases de dados nacionais, no período de 2015 a 2020. Resultado: Foram selecionados cinco artigos destinados a analisar, avaliar, verificar e investigar o conhecimento (teoria) da equipe de enfermagem, na hemotransfusão; dois estudos procuraram elaborar e descrever tecnologias educacionais voltados para o ensino de enfermagem na assistência ao processo de transfusão sanguínea, destes um estudo descreveu O processo de construção e validação de conteúdo de um checklist para transfusão sanguínea em crianças, e o outro elaborou um Instrumento para a monitorização do paciente submetido à transfusão de sangue. A partir dos resultados encontrados, foram formadas quatro categorias temáticas: O momento da pré transfusão: passo ouro inicial; O momento da transfusão; O momento da pós transfusão: ainda não é o final e as Ferramentas de gerenciamento de risco na hemotransfusão. Conclusão: Ressaltamos que apesar da equipe de enfermagem relatar terem segurança nas etapas, porém é contraditório aos dados fornecidos nos estudos como a incipiência do conhecimento da equipe de enfermagem na hemotransfusão; esses dados respondem à questão da pesquisa e corroboram com os objetivos desse estudo.
\end{abstract}

Palavras-chave: Hemotransfusão; Equipe de enfermagem; Conhecimento.

\begin{abstract}
Objective: To identify scientific studies on the knowledge of the nursing team in the face of a blood transfusion; observe the degree of knowledge of the nursing team regarding blood transfusion; identify the risk management tools that can contribute to this knowledge. Methodology: It is an Integrative Literature Review, exploratory with a qualitative approach on the knowledge of the nursing team on blood transfusion, carried out in national databases, in the period from 2015 to 2020 . Result: Five articles were selected to analyze, evaluate, verify and investigate the knowledge (theory) of the nursing team, in blood transfusion; two studies sought to develop and describe educational technologies aimed at teaching nursing in assisting the blood transfusion process, of these one study described the process of construction and content validation of a checklist for blood transfusion in children, and the other developed an Instrument for monitoring of the patient undergoing blood transfusion. From the results found, four thematic categories were formed: The moment of pre-transfusion: initial gold step; The moment of transfusion; The moment of post-transfusion: it is not yet the end and the risk management tools in blood transfusion. Conclusion: We emphasize that although the nursing team reports having safety in the stages, it is contradictory to the data provided in the studies with the incipience of the knowledge of the nursing team in blood transfusion; these data answer the research question and corroborate the objectives of this study.
\end{abstract}

Keywords: Blood transfusion; Nursing team; Knowledge.

\title{
Resumen
}

Objetivo: Identificar estudios científicos sobre el conocimiento del equipo de enfermería ante una transfusión de sangre; observar el grado de conocimiento del equipo de enfermería con respecto a la transfusión de sangre; Identificar las herramientas de gestión de riesgos que pueden contribuir a este conocimiento.Metodología: Se trata de 
una Revisión de Literatura Integrativa, exploratoria con abordaje cualitativo sobre los conocimientos del equipo de enfermería sobre transfusión sanguínea, realizada en bases de datos nacionales, en el período de 2015 a 2020.Resultado: Se seleccionaron cinco artículos para analizar, evaluar, verificar e investigar el conocimiento (teoría) del equipo de enfermería, en transfusión sanguínea; dos estudios buscaron desarrollar y describir tecnologías educativas dirigidas a la enseñanza de enfermería en la asistencia al proceso de transfusión sanguínea, de estos un estudio describió el proceso de construcción y validación de contenido de una lista de verificación para transfusión sanguínea en niños, y el otro desarrolló un Instrumento para el seguimiento de la el paciente que se somete a una transfusión de sangre. A partir de los resultados encontrados se formaron cuatro categorías temáticas: El momento de la pretransfusión: paso inicial de oro; El momento de la transfusión; El momento de la postransfusión: aún no es el final y las herramientas de gestión de riesgos en la transfusión de sangre.Conclusión: Destacamos que si bien el equipo de enfermería reporta tener seguridad en las etapas, sin embargo, es contradictorio con los datos proporcionados en los estudios, como la incipiencia del conocimiento del equipo de enfermería en transfusión sanguínea; estos datos responden a la pregunta de investigación y corroboran los objetivos de este estudio.

Palabras clave: Transfusión de sangre; Equipo de enfermería; Conocimiento.

\section{Introdução}

Hemoterapia é o emprego terapêutico do sangue nos cuidados a saúde. Essa prática terapêutica vem sendo estudada há décadas, passando por vários momentos, evoluindo rapidamente em termos científico e apresentando uma grande perspectiva futura. As bolsas ou unidades de sangue total são coletadas de doadores voluntários, encaminhadas aos centros especializados (hemocentros) onde serão centrifugadas e fracionadas para obtenção dos hemocomponentes sanguíneos, tais como: concentrado de hemácias, concentrado de plaquetas e plasma fresco congelado, ou seja, a partir de cada bolsa doada, são produzidos diversos produtos (hemocomponentes) e os derivados (hemoderivados como fatores de coagulação, albumina, entre outros) (Silva, Assis \& Silva, 2017).

A segurança na transfusão e a gestão da qualidade estão diretamente relacionadas entre si, haja vista que, qualidade nos serviços de saúde significa oferecer menor risco ao paciente, a partir da instrumentalização, sistematização e a busca da maximização dos cuidados e dos benefícios. Para isto, o estabelecimento de um planejamento e de uma política contínua de gerenciamento de riscos, como, por exemplo, protocolos consolidados na organização, contribuem para a segurança e beneficiam as partes interessadas: o paciente, o colaborador e a instituição (Mattia \& Andrade, 2016).

Uma hemorragia com risco de vida, que requer transfusão maciça pode ocorrer em uma variedade de situações, incluindo hemorragias intra e pós-operatórias inesperadas, bem como em lesões traumáticas, dentre outras condições clínicas (Langston et al. 2017).

No entanto a hemotransfusão não é isenta de riscos. Podem acontecer reações de diversas naturezas, as quais são classificadas em imediatas ou tardias. De acordo com a RDC n ${ }^{\circ}$ 153/2004 da Agência Nacional de Vigilância Sanitária (ANVISA), as reações imediatas ocorrem durante a transfusão ou em até 24 horas após o procedimento. As tardias acontecem depois de 24 horas da transfusão. Os incidentes transfusionais imediatos dividem-se em: reação hemolítica aguda, reação febril não hemolítica, reação alérgica leve, moderada e grave, sobrecarga volêmica, contaminação bacteriana, edema pulmonar não cardiogênico (TRALI), reação hipotensiva e hemólise não imune, os quais devem ser notificados aos serviços de controle de qualidade em hemoterapia (Carneiro; Barp \& Coelho, 2017).

Os profissionais de saúde devem entender os possíveis impactos das transfusões de hemocomponentes e hemoderivados, e os processos que envolvem a terapia de transfusão para garantir assistência de alta qualidade, resultados positivos e uso inteligente de recursos. A compreensão das transfusões de sangue e hemoderivados também é importante devido ao número de tratamentos realizados, o que afeta os pacientes individualmente e os recursos do sistema de saúde (Passerin, 2019). Os conhecimentos e habilidades dos profissionais de saúde são fundamentais para o desenvolvimento e fortalecimento da qualidade dos procedimentos de transfusão sanguínea (Encan \& Akin, 2019). 
Os profissionais de enfermagem, em suas três categorias, detêm a responsabilidade pela administração de transfusões de sangue, e o fazem com grande frequência. No Brasil, as competências e atribuições do enfermeiro e técnicos em enfermagem em hemoterapia são regulamentadas pela Resolução no 511/2016 do Conselho Federal de Enfermagem, a qual estabelece a sua responsabilidade pelo planejamento, execução, coordenação, supervisão e avaliação de procedimentos de hemoterapia nas unidades de saúde (Silva et al. 2017).

A equipe de enfermagem, por permanecer à frente da assistência durante as 24 horas do dia, exerce papel fundamental na terapia transfusional. Os profissionais, não apenas realizam a administração de componentes e derivados sanguíneos, mas também providenciam a checagem de dados importantes na prevenção de erros, orientam os pacientes sobre a transfusão, detectam, comunicam e atuam no atendimento das reações transfusionais e documentam todo o processo (Carneiro; Barp \& Coelho, 2017).

A hemotransfusão deve ser executada por profissionais qualificados e com habilidades e competências técnicas específicas, em condições e ambiente seguro para atender possíveis intercorrências, para que, desta forma, seja assegurada a qualidade do cuidado (Silva, Assis \& Silva, 2017). Apesar dos avanços da hemovigilância no Brasil, ainda há muitos desafios a serem superados na busca da qualidade da assistência hemoterápica para a redução do risco à saúde. A ausência de programas de educação continuada voltados para à equipe de enfermagem é uma realidade, sendo necessária a promoção de educação permanente que vise ao desenvolvimento de conhecimentos, habilidades e competências da equipe de enfermagem em seu cotidiano nos setores de terapia intensiva e urgência/ emergência (Silva, Vieira, Silva \& Ferreira, 2017).

Diante do exposto formulou - se a seguinte questão: Quais as evidências científicas sobre o Conhecimento da Equipe de Enfermagem sobre o processo de hemotransfusão?

Contribuindo com a praxis da assistência de enfermagem, este estudo tem como objetivos: Identificar os estudos científicos sobre o conhecimento da equipe de enfermagem diante de uma transfusão sanguínea; observar qual o grau de conhecimento da equipe de enfermagem referente à hemotransfusão; identificar as ferramentas de gerenciamento de risco que possam contribuir para esse conhecimento.

\section{Método}

Optou-se por realizar revisão integrativa, que se constitui na mais ampla abordagem metodológica referente às revisões, reunindo de forma sistemática e rigorosa achados de estudos desenvolvidos mediante diferentes metodologias, buscando uma compreensão completa do fenômeno analisado (Vieira \& Santos, 2020).

Para elaboração deste estudo foram seguidas as seis fases do processo de elaboração da revisão integrativa: elaboração da pergunta norteadora; busca ou amostragem na literatura; coleta de dados; análise crítica dos estudos incluídos; discussão dos resultados; apresentação da revisão integrativa (Costa et al. 2019).

Para a coleta de dados, foi adaptado um instrumento desenvolvido por Ursi (Ursi \& Galvão,2006). A busca foi realizada com o emprego do DeCS/MeSH, blood transfusion AND nursing (transfusão sanguínea E enfermagem), nas bases de dados: Biblioteca Virtual em Saúde (BVS), National Library of Medicine (PubMed), Scielo e Biblioteca Digital Brasileira de Teses e Dissertações da Coordenação de Aperfeiçoamento de Pessoal de Nível Superior (CAPES) no mês de novembro de 2020.

Os critérios para inclusão foram: estudos brasileiros, cujo objeto era o conhecimento dos profissionais de enfermagem em transfusão de sangue, publicados entre 2015 e 2020, no idioma português. No processo de exclusão a linha de descarte, foram estudos brasileiros que envolviam outros profissionais e estudantes na graduação, além de estudos estrangeiros. É importante frisar que a escolha do período de 5 anos, é para que possamos ter a visibilidade de artigos com publicação recente, mostrando a importância desse estudo. 
No que tange à avaliação das evidências oriundas dos estudos selecionados, optou-se pela classificação proposta por Stetler e colaboradores, a saber: Nível I - Metanálisee múltiplos estudos controlados; Nível II - Estudos de desenho experimental; Nível III - Estudos de desenho quase-experimental; Nível IV - Estudos de desenho nãoexperimental ou de natureza qualitativa e, ainda, os estudos de caso; Nível V - Relatos de experiência ou relatórios de caso; Nível VI- Opinião de autoridades respeitadas baseada em sua experiência clínica ou opinião de um comitê de especialistas (Vieira \& Santos, 2020).

\section{Resultado}

A busca preliminar resultou em 7 publicações na BVS, 9 na PubMed, 28 Scielo e 4 estudos na Biblioteca Digital Brasileira de Teses e Dissertações da CAPES. Realizou-se a leitura dos resumos e, posteriormente, a seleção para leitura dos textos completos: na BVS selecionaram-se quatro artigos, na PubMed três, na Scielo foram selecionados dez, e na Biblioteca Digital Brasileira de Teses e Dissertações da CAPES dois estudos foram selecionados. Após a leitura dos textos completos, houve o descarte de onze estudos que não atendiam aos critérios de inclusão sendo: dois estudos com idioma em inglês da BVS, um estudo da CAPES em repetição com a Scielo, um estudo da PubMed em repetição com a Scielo, dois estudos da PubMed (um estrangeiro e o outro não contempla o assunto em questão), cinco estudos da Scielo (três estrangeiros, um não contempla o assunto em questão e um refere - se a outras categorias profissionais).

Dos artigos que constituíram esta revisão integrativa da literatura, cinco destinados a analisar, avaliar, verificar e investigar o conhecimento (teoria) da equipe de enfermagem, na hemotransfusão; dois estudos procuraram elaborar e descrever tecnologias educacionais voltados para o ensino de enfermagem na assistência ao processo de transfusão sanguínea, destes um estudo descreveu e elaborou O processo de construção e validação de conteúdo de um checklist para transfusão sanguínea em crianças, e o outro elaborou um Instrumento para a monitorização do paciente submetido à transfusão de sangue.

Em relação ao ano de publicação, houve um artigo (9,09\%) em 2015, dois (18,18\%) em 2016, dois $(18,18 \%)$ em 2017 e dois $(18,18 \%)$ em 2018. Ressaltamos que os estudos são (100\%) desenvolvidos no Brasil. Sete estudos (100\%) foram publicados em Revista de Enfermagem. Todos os artigos são originais, encontrados nas bases de dados relatados acima. Dois artigos com abordagem metodológica (18,18\%), um artigo observacional, transversal quantitativo (9,09\%), um descritivo com abordagem quantitativa $(9,09 \%)$, dois descritivos, estudos exploratórios com abordagem qualitativa (18,18\%), um artigo com abordagem qualitativa $(9,09 \%)$ e por fim um artigo descritivo, retrospectivo com abordagem quantitativa $(9,09 \%)$. O Quadro 1 mostra a síntese dos artigos segundo autor, título, periódico, ano, procedimento metodológico e resultados da pesquisa.

Quadro 1 - Síntese dos artigos segundo autor, título, periódico, ano, procedimento metodológico e resultados da pesquisa.

\begin{tabular}{|c|c|c|c|c|}
\hline Código & Autor, Título, Periódico, Ano. & Base de Dados & $\begin{array}{l}\text { Procedimento } \\
\text { Metodológico }\end{array}$ & Resultado da Pesquisa \\
\hline A1 & $\begin{array}{l}\text { Tavares, JL, Barrichello, E, Mattia, } \\
\text { A.L.de, Barbosa, M.H. } \\
\text { Fatores associados ao } \\
\text { conhecimento da equipe de } \\
\text { enfermagem de um hospital de } \\
\text { ensino sobre hemotransfusão. } \\
\text { Revista Latino-Americana } \\
\text { de Enfermagem, } 2015\end{array}$ & $\begin{array}{l}\text { Web of } \\
\text { Science }\end{array}$ & $\begin{array}{l}\text { Estudo } \\
\text { observacional, } \\
\text { transversal, } \\
\text { quantitativo. }\end{array}$ & $\begin{array}{l}\text { A média de escore geral de conhecimento foi de } 52,66 \% \text {, } \\
\text { Os fatores relacionados ao conhecimento foram categoria } \\
\text { profissional e receber treinamento e/ou orientação para a } \\
\text { realização do processo transfusional (p\&lt;0,01). Este } \\
\text { estudo evidenciou a influência do treinamento e } \\
\text { orientação sobre o conhecimento e forneceu um } \\
\text { diagnóstico para a identificação das dificuldades dos } \\
\text { profissionais relacionadas ao processo transfusional. }\end{array}$ \\
\hline A2 & $\begin{array}{l}\text { Mattia D, Andrade SR de. } \\
\text { Cuidados de enfermagem na } \\
\text { transfusão de sangue: um } \\
\text { instrumento para monitorização do } \\
\text { paciente. Texto contexto - }\end{array}$ & Scielo & $\begin{array}{l}\text { Estudo } \\
\text { abordagem } \\
\text { qualitativa. }\end{array}$ & $\begin{array}{l}\text { Identificou-se que os profissionais compreendem o } \\
\text { conceito de qualidade e procuram implementar ações para } \\
\text { alcançar um padrão ótimo de cuidado ao paciente. Em } \\
\text { consonância com a norma vigente, os profissionais } \\
\text { estruturaram um instrumento que permitirá o registro das }\end{array}$ \\
\hline
\end{tabular}




\begin{tabular}{|c|c|c|c|c|}
\hline & enferm. 2016. & & & $\begin{array}{l}\text { informações sobre a transfusão de sangue, servindo como } \\
\text { ferramenta para monitorar o paciente submetido a essa } \\
\text { terapêutica. }\end{array}$ \\
\hline A3 & $\begin{array}{l}\text { Reis VN dos, Paixão IB, Perrone } \\
\text { ACSJ, Maria MI, Santos KB dos. } \\
\text { Monitorização transfusional: } \\
\text { análise da prática assistencial em } \\
\text { um hospital público de ensino. } \\
\text { Einstein (São Paulo) } \\
2016 .\end{array}$ & Scielo & $\begin{array}{l}\text { Estudo } \\
\text { descritivo, } \\
\text { retrospectivo, de } \\
\text { abordagem } \\
\text { quantitativa. }\end{array}$ & $\begin{array}{l}\text { Foram analisados } 1.012 \text { instrumentos, dos quais } 53,4 \% \\
\text { apresentaram falhas no preenchimento, } 6 \% \text { das infusões } \\
\text { foram iniciadas após o tempo preconizado e } 9,3 \% \text { dos } \\
\text { pacientes não tiveram os sinais vitais registrados. Foram } \\
\text { identificadas falhas no processo de registro da } \\
\text { monitorização transfusional, que podem gerar maior } \\
\text { ocorrência de eventos adversos relacionados à } \\
\text { administração de hemocomponentes. }\end{array}$ \\
\hline A4 & $\begin{array}{l}\text { Silva EM, Vieira CA, Silva FO, } \\
\text { Ferreira EV. Desafios da } \\
\text { enfermagem diante das reações } \\
\text { transfusionais. Rev. enferm. UERJ. } \\
2017\end{array}$ & $\begin{array}{l}\text { Biblioteca } \\
\text { Virtual em } \\
\text { Saúde (BVS). }\end{array}$ & $\begin{array}{l}\text { Pesquisa de } \\
\text { caráter descritivo } \\
\text { com abordagem } \\
\text { quantitativa. }\end{array}$ & $\begin{array}{l}\text { Entre os entrevistados, } 93 \% \text { foram mulheres, } 80 \% \text { com } \\
\text { tempo de formação superior a } 10 \text { anos, das quais } 49 \% \\
\text { nunca monitoraram transfusões de hemocomponentes ou } \\
\text { hemoderivados. Verificou- se que } 59 \% \text { desconhecem o } \\
\text { tempo máximo para a infusão do concentrado de hemácias } \\
\text { e } 76 \% \text { não sabiam o tempo mínimo para realizar essa } \\
\text { infusão. Quanto às reações adversas, } 65 \% \text { afirmaram saber } \\
\text { identificá-las e } 19 \% \text { não souberam a conduta que deviam } \\
\text { adotar diante de reações transfusionais. }\end{array}$ \\
\hline A5 & $\begin{array}{l}\text { Cherem EO, AlvesVH, Rodrigues } \\
\text { DP, Souza FDL, } \\
\text { GuerraJVV, Maciel VL. Saberes } \\
\text { do enfermeiro para o cuidado no } \\
\text { processo transfusional em recém- } \\
\text { nascidos. Rev Gaúcha } \\
\text { Enfermagem } 2017\end{array}$ & Pubmed/Scielo. & $\begin{array}{l}\text { Estudo descritivo } \\
\text { e exploratório } \\
\text { qualitativo. }\end{array}$ & $\begin{array}{l}\text { A categoria criada foi dividida nas seguintes } \\
\text { subcategorias, a saber: cuidados do enfermeiro durante a } \\
\text { hemotransfusão: cuidados durante o acompanhamento da } \\
\text { hemotransfusão; uma percepção diferente dos enfermeiros } \\
\text { quanto às respostas à transfusão sanguínea e notificação: a } \\
\text { essência do cuidado. A identificação do conhecimento dos } \\
\text { enfermeiros sobre hemotransfusão, é possível promover } \\
\text { sua capacidade para a qualidade e segurança da assistência } \\
\text { relacionada ao processo transfusional do recém-nascido. }\end{array}$ \\
\hline A6 & $\begin{array}{l}\text { Bezerra CM, Cardoso MVLML } \\
\text { Silva GRF, Rodrigues EC. } \\
\text { Criação e validação de uma lista de } \\
\text { verificação para transfusão de } \\
\text { sangue em crianças. Rev Bras } \\
\text { Enfermagem } \\
2018\end{array}$ & Pubmed/Scielo. & $\begin{array}{l}\text { Estudo } \\
\text { Metodológico. }\end{array}$ & $\begin{array}{l}\text { O conteúdo foi considerado válido com IVC global de } \\
0,87 \text {. Sugestões de ajustes, como exclusão, substituição e } \\
\text { acréscimo de termos, foram incluídos na versão final, que } \\
\text { era composta por } 14 \text { itens e } 56 \text { subitens. O checklist para } \\
\text { transfusão de sangue em crianças foi considerada uma } \\
\text { tecnologia com conteúdo válido para ser utilizada na } \\
\text { transfusão realizada por enfermeiros, contribuindo assim } \\
\text { para a segurança transfusional em crianças. }\end{array}$ \\
\hline A7 & $\begin{array}{l}\text { Cherem EO, Alves VH, } \\
\text { Rodrigues DP; Pimenta PCO, } \\
\text { Souza FDL, Guerra } \\
\text { JVV. Processo de terapia } \\
\text { transfusional em unidade de terapia } \\
\text { intensiva neonatal: o conhecimento } \\
\text { do enfermeiro. } \\
\text { Texto contexto enferm. } 2018\end{array}$ & Scielo & $\begin{array}{l}\text { Estudo } \\
\text { descritivo, } \\
\text { exploratório, } \\
\text { qualitativo. }\end{array}$ & $\begin{array}{l}\text { O processo pré-transfusional na assistência ao recém- } \\
\text { nascido e sua interface para a assistência obteve duas } \\
\text { subcategorias: Contextualizando a prática na indicação } \\
\text { transfusional e nos exames de compatibilidade sanguínea; } \\
\text { e cuidados de enfermagem ao recém-nascido antes do } \\
\text { procedimento transfusional. Com isso, as enfermeiras } \\
\text { citaram a importância de conferir a bolsa de sangue, } \\
\text { puncionar o acesso venoso periférico e não administrar } \\
\text { antibióticos no momento da infusão. }\end{array}$ \\
\hline
\end{tabular}

Fonte: Autores.

\section{Discussão}

A partir dos resultados, separaram-se as temáticas em três categorias, discutidas e detalhadas a seguir. 


\section{Categoria 1- O momento pré-transfusional: passo ouro inicial}

Em estudo com 209 profissionais da equipe de enfermagem (artigo A1); na etapa pré-transfusional, a média de conhecimento foi de $53,38 \%$; porém neste mesmo estudo foi observado que $92,8 \%$ dos profissionais sentem-se seguros para a realização do processo transfusional. Em estudo realizado na França, 83\% dos profissionais de enfermagem disseram sentir-se bem informados com relação à transfusão. Outras pesquisas apontaram que, em média, $60 \%$ dos profissionais referiram sentirse informados sobre a prática transfusional (Tavares et al, 2015).

Estes achados apesar de mostrarem dados aceitáveis de conhecimento por parte da equipe de enfermagem corroboram com o objetivo da pesquisa, da necessidade desta equipe está capacitada no que se refere ao processo de hemotranfusão, pois como revelado em "sentem-se seguros para a realização do processo transfusional", não significa que detem o conhecimento do processo transfusional em sua integra.

Neste mesmo estudo os autores demonstram que em Mali, na África Ocidental, evidenciou que 53,9\% dos participantes apresentaram conhecimento insuficiente e que o grupo de enfermeiras e de parteiras apresentou mais inadequações no conhecimento. Em pesquisa realizada com enfermeiros em Shahrekord, no Irã, concluiu-se que o conhecimento das indicações e complicações da transfusão de sangue dos enfermeiros foi médio, visto que 16,2\% tinham bom nível de conhecimento, 59\% tiveram médio e 24,8\% tinham baixo nível de conhecimento (Tavares et al, 2015).

Evidencia-se nesses estudos, que os dados são ainda mais preocupantes, pois os profissionais de enfermagem desenvolvem uma prática no processo da hemotransfusão totalmente desprovidos de conhecimento, mostrando que os estudos estrangeiros pouco diferenciam a estudos brasileiros, na clareza da falta de conhecimento da equipe de enfermagem nesse processo.

Em pesquisa realizada com profissionais de enfermagem de um Hospital Universitário, especificamente na agência transfusional (artigo A2), os participantes evidenciaram que com relação aos dados pré-transfusionais, abordou-se necessidade de apresentar, além dos sinais vitais (pressão arterial, frequência cardíaca, frequência respiratória e temperatura), data e hora de início da transfusão, via de acesso (periférico, central, port cath), local do acesso, dispositivo utilizado (único, compartilhado), orientação do paciente ou responsável sobre o procedimento e campo para observações. Neste mesmo estudo mostrou que no Reino Unido, identificou que, aproximadamente, $66,7 \%$ das reações transfusionais notificadas naquele país estão associadas a erros de identificação de receptores. Tal fato evidencia a importância de contemplar estas informações em instrumento de monitorização (Matias \& Andrade, 2016).

Para os a que a maioria dos setores do hospital estudado apresentou os instrumentos preenchidos de forma inadequada, faltando dados como nome da mãe do paciente, peso, número do prontuário, setor, leito, horário de início e término da transfusão, e assinatura do responsável pelo ato transfusional.

Este resultado difere do que é proposto pela World Heath Organization (WHO), que preconiza que a orientação clínica para transfusão de sangue e hemoderivados deve contemplar a identificação positiva dos pacientes antes da transfusão. Pesquisa realizada com enfermeiras sobre seu conhecimento em hemotransfusão em dois hospitais de Abu Dhabi, capital dos Emirados Árabes Unidos, identificou baixos índices em diversos pontos chaves do processo.

Merece destaque o fato de que somente $36 \%$ detinham o conhecimento das ações a serem tomadas antes de iniciar a transfusão, como identificação do paciente e aferição dos sinais vitais, e apenas 19\% conheciam a classificação ABO. Em estudo francês, apenas $25 \%$ dos enfermeiros $(n=42)$ tinham conhecimento apropriado sobre a prática hemoterápica segura, principalmente relacionada à checagem da compatibilidade pré-transfusional (30\%), reconhecimento de reações após a transfusão (40\%) e demora na preservação do hemocomponente na enfermaria (65\%) (Reis et al., 2016).

Na pesquisa com 35 enfermeiros de duas UTINs (artigo A8), mostrou-se que um aspecto que merece destaque é que uma requisição incompleta, inadequada ou ilegível deve ser avaliada pelo enfermeiro, até porque para que ocorra a transfusão, 
ele precisa conferir esse pedido de sangue, que deve ser preenchido corretamente, já que se constitui em via documental legal e justifica a necessidade desse procedimento (Cherem et al., 2018.).

O que podemos perceber nas pesquisas, que a realização da conferência de todos os dados na pré transfusão está sendo negligenciada pela equipe de enfermagem, mostrando lacunas para o desenvolvimento da etapa seguinte da aplicação do sangue de forma tranquila, segura e sem riscos ao paciente.

\section{Categoria 2- O momento transfusional: à hora do conhecimento}

No estudo com profissionais de enfermagem (artigo A2) no tópico relacionado ao período de transfusão, os participantes elencaram como necessário conter o registro dos sinais vitais. Esses dados permitem monitorar o paciente no decorrer da transfusão e identificar precocemente possíveis reações transfusionais, visto que é uma terapêutica que não está isenta de causar dano à saúde; e a equipe de enfermagem necessita ter o conhecimento sobre essa possível intercorrência e identificar os sinais e sintomas precocemente, pois isso poderá determinar o tipo de reação transfusional e a tomada de decisão para a conduta terapêutica (Matias \& Andrade, 2016).

Importante contribuição neste estudo (artigo A3), onde um grande número de fichas não continha o registro do monitoramento dos sinais vitais durante a transfusão, o que permite inferir que há possibilidade dessa etapa estar sendo negligenciada por alguns profissionais da instituição. Evidências demonstram que a importância da monitorização da transfusão reside no fato de ela permitir o reconhecimento e a mitigação ativa, oportuna e precoce, bem como a correção de efeitos fisiopatológicos e de reações transfusionais graves, como a reação hemolítica aguda e a lesão pulmonar aguda associada à transfusão (TRALI - transfusion-related acute lung injury). (Reis et al., 2016).

Pode-se inferir que não só o acompanhamento dos sinais vitais na hora da transfusão sanguínea é suficiente para corrigir e salva vida, mas o conhecimento acerca do reconhecimento de reações adversas e tomada de medidas preventivas imediatas.

A pesquisa realizada com 95 profissionais de enfermagem (artigo A4) identificaram os sinais apresentados durante uma reação transfusional, foram os mais citados pelos participantes deste estudo febre, tremores e taquicardia. Porém, observou-se que a equipe de enfermagem pesquisada desconhecia grande parte dos sinais apresentados pelos pacientes. Nesse mesmo estudo, mostra que em um hospital universitário do interior de São Paulo, que buscou determinar o conhecimento das práticas transfusionais por parte dos profissionais de enfermagem, verificou que nem sempre os profissionais que atuam na hemoterapia estão habilitados para atuar no processo transfusional e no atendimento às suas reações adversas (SILVA et al. 2017).

Em consonâncias com os estudos apresentados, revelam que os profissionais da equipe de enfermagem, precisam constantemente de atualizações, capacitações para a realização de transfusão sanguínea segura.

\section{Categoria 3- O momento pós-tranfusional: ainda não é o final}

Neste estudo (artigo A2) quanto ao tópico associado ao período pós transfusão, os participantes apontaram a importância de manter registrados os sinais vitais, hora de término da transfusão, remoção ou não do acesso utilizado para a transfusão, campo para observações a fim de descrever possíveis reações adversas e condutas. Esses dados são importantes, pois permitem observar se a transfusão ocorreu dentro do tempo determinado pela legislação (4 horas no máximo) e a identificação de uma possível reação transfusional. O tempo de administração do hemocomponente é fundamental, pois caso seja ultrapassado, o hemocomponente perde suas propriedades pela exposição à temperatura não controlada. Isso pode acarretar também a elevação do risco para o crescimento bacteriano. Neste mesmo estudo demonstram que as reações transfusionais que ocorrem durante e até 24 horas após a transfusão de sangue ainda representam quase que a totalidade das 
reações transfusionais notificadas. Isso demonstra a necessidade de monitorar o paciente durante todo o processo de transfusão (Matias \& Andrade, 2016).

Estudo realizado em um hospital na cidade do Recife mostrou que $71 \%$ dos profissionais de enfermagem não sabem que conduta tomar diante de reações tardias ou mediatas. Estes estudos corroboram com a importância da equipe de enfermagem está devidamente capacitada para atuar nas três etapas do processo transfusional, principalmente frente às reações transfusionais, mesmo após o término da hemotransfusão e até 24h após a retirada da bolsa de sangue.

\section{Categoria 4- Ferramentas de gerenciamento de risco na hemotransfusão}

Mattias e Andrade (2016) apontam que o registro das informações relacionadas à transfusão de sangue é de extrema importância, pois serve para verificar se a transfusão ocorreu de acordo com as normas vigentes. Assim o monitoramento de todo o processo transfusional objetiva detectar queixas, sinais e sintomas que possam evidenciar reações transfusionais. O registro e o acompanhamento dos eventos adversos associados à transfusão, e contribuem para a garantia da segurança e o controle da qualidade dos serviços de hemoterapia.

Este mesmo estudo, na fala de enfermeiros participantes de pesquisa (artigo A2) nos grupos de discussão foi sendo estruturado um instrumento para a monitorização do paciente submetido à transfusão de sangue. Utilizando perguntas norteadoras, foram elencados tópicos que devem estar presentes no instrumento e os conteúdos necessários que cada tópico deve apresentar.

Estudo realizado com 52 enfermeiros e 79 técnicos de enfermagem em um hospital universitário público, no estado do Rio de Janeiro, realizou em sua Fase II - Elaboração da Lista de Verificação Segura para o Cliente, essa Lista de Verificação (Checklist) Seguro para a Assistência de Enfermagem ao Cliente Receptor de Hemocomponentes instrumentaliza a assistência de enfermagem e seu preenchimento alimenta os relatórios gerenciais permitindo o monitoramento da assistência de enfermagem em hemoterapia realizado pelos enfermeiros e técnicos de enfermagem. O seu preenchimento deverá ser realizado a cada transfusão de hemocomponente (Souza, 2017).

No estudo de Bezerra et al. (2016) (artigo A7) de posse dos conteúdos elencados pelo levantamento bibliográfico, foi iniciado o processo de construção. O checklist construído foi do tipo "leia-faca", no qual existe uma lista de tarefas a serem executadas, geralmente, por uma pessoa. O instrumento baseou-se no layout e nos princípios de simplicidade, ampla aplicabilidade e possibilidade de mensuração do Checklist para Segurança Cirúrgica da Organização Mundial de Saúde. O checklist construído para transfusão sanguínea em crianças, semelhante ao Checklist da Cirurgia Segura da OMS, divide o procedimento em três momentos (etapa pre-transfusional, transfusional e pos-transfusional).

Podemos observar que mesmo com informações preocupantes no que tange ao conhecimento da equipe de enfermagem sobre o processo de transfusão sanguínea em suas três etapas; verificamos que assim como as autoras deste artigo, outros autores manifestam a sensibilidade de construir e validar instrumentos que possam contribuir na prática da equipe de enfermagem frente à hemotransfusão segura sem riscos para o paciente.

\section{Conclusão}

Dos sete estudos encontrados nesta revisão da literatura, todos trataram sobre o conhecimento da equipe de enfermagem nas etapas de hemotranfusão, dentre esses, dois elaboraram instrumentos como ferramenta de gerenciamento de risco para ser aliada no referido processo. Ressaltamos que apesar da equipe de enfermagem relatar terem segurança nas etapas, é contraditório aos dados fornecidos nos estudos com a incipiência do conhecimento da equipe de enfermagem na hemotransfusão; esses dados respondem a questão da pesquisa e corroboram com os objetivos desse estudo. 
Neste mesmo contexto reafirmamos a importância de se fazer investimentos na área de atualizações e capacitações, além de treinamento para as equipes iniciantes nessa atividade; além de averiguar e ampliar melhor suporte teórico/técnico nas grades curriculares dos cursos de formação técnica e de graduação. Reafirmamos também a importância da construção e validação de instrumentos para monitorização e auxiliar a equipe de enfermagem nas etapas transfusionais.

Como limitação da pesquisa, os participantes desta revisão da literatura foram avaliados através de questionários, ficando uma lacuna na observação desses profissionais em sua rotina de trabalho.

As autoras sugerem que sejam realizadas avaliações teóricas aliadas as práticas diárias em outras pesquisas, assim poderão compreender melhor quando a equipe relatar ter segurança em realizar as etapas transfusionais verso a deficiência do conhecimento teórico do assunto.

\section{Referências}

Agência Nacional de Vigilância Sanitária (2011). Boletim de hemovigilância, Ministério da Saúde. https://www.scielo.br/scielo.php?pid=S0034$71672020001500176 \&$ script=sci_arttext\&tlng=pt.

Agência Nacional de Vigilância Sanitária (2011). Resolução n ${ }^{\circ} 113$ de 14 de junho de 2011 . Regulamento técnico de procedimentos hemoterápicos. ANVISA. http://dspace.mj.gov.br/bitstream/1/897/2/PRT_SNJ_2011_13.pdf.

Brasil. Conselho Federal de Enfermagem. Resolução COFEN no 564/2017. Aprova o novo código de ética dos profissionais de enfermagem. Brasília. 6 de novembro de 2017. http://biblioteca.cofen.gov.br/wp-content/uploads/2017/07/C\%C3\%B3digo-de-\%C3\%89tica-dos-Profissionais-de-Enfermagem-umapesquisa-documental.pdf.

Bezerra, C. M., Cardoso, M. V. L. M. L., Silva, G. R. F., \& Rodrigues, E. C. (2018). Criação e validação de uma lista de verificação para transfusão de sangue em crianças. Rev Bras Enfermagem. https://www.scielo.br/scielo.php?pid=S0034-71672018000603020\&script=sci_arttext\&tlng=pt.

Conselho Nacional de Saúde (2012). Resolução no 466 de junho de 2012. Diretrizes e normas regulamentadoras de pesquisas envolvendo seres humanos. Brasília (DF): CNS. https://www.scielosp.org/article/sausoc/2019.v28n4/299-310/.

Costa, T. R. M., Santos, S. F. D., Rocha, P. R. M, Pinto. A. R., Nogueira. M. A., \& Ingrid Pimentel, I. M. S., (2019). Cuidados de enfermagem na prevenção da ceratopatia por exposição na unidade de terapia intensiva. Enfermagem Brasil,18(3):453-459. Recuperado em: https://portalatlanticaeditora.com.br.

Cherem, E. O., Alves, V. H., Rodrigues, D. P., Souza, F. D. L., Guerra, J. V. V., \& Maciel V. L. (2017). Saberes do enfermeiro para o cuidado no processo transfusional em recém-nascidos. 38(1). Rev Gaúcha Enfermagem. https://www.scielo.br/scielo.php?pid=S1983$14472017000100411 \&$ script=sci_arttext\&tlng=pt.

Cherem, E. O., Alves, V. H., Rodrigues, D. P., Pimenta, P. C. O., Souza, F. D. L., \& Guerra, J. V. V. (2018). Processo de terapia transfusional em unidade de terapia intensiva neonatal: o conhecimento do enfermeiro. Texto contexto enferm. https://www.scielo.br/scielo.php?pid=S0104$07072018000100301 \&$ script=sci_arttext\&tlng=es

Duarte, R. D., Silva, K. F. N., Félix, M. M. S., Tavares, J. L., Zuffi, F. B., \& Barbosa, M. H. (2017). Conhecimento sobre hemotransfusão em unidade crítica de hospital de ensino. Biosci. J. https://www.redalyc.org/pdf/2814/281442224005.pdf

Mattia, D., \& Andrade, S. R. (2016). Cuidados de enfermagem na transfusão de sangue: um instrumento para monitorização do paciente. Texto contexto enferm. 25(2). https://www.scielo.br/scielo.php?pid=S0104-07072016000200308\&script=sci_arttext\&tlng=pt

Ministério da Saúde (2010). Guia para o uso de hemocomponentes. Brasília (DF): Ministério da Saúde. http://bvsms.saude.gov.br/bvs/publicacoes/guia_uso_hemocomponentes.pdf.

Reis, V. N., Paixão, I. B., Perrone, A. C. S. J., Maria, M. I., \& Santos, K. B. (2016) Monitorização transfusional: análise da prática assistencial em um hospital público de ensino. Einstein. https://journal.einstein.br/pt-br/article/monitorizacao-transfusional-analise-da-pratica-assistencial-em-um-hospital-publico-deensino/.

Silva, K. F. N., Soares, S., \& Iwamoto, H. H. (2009). A prática transfusional e a formação dos profissionais de saúde. Rev. Bras. Hematol. Hemoter. https://www.scielo.br/scielo.php?pid=S1516-84842009005000092\&script=sci_arttext.

Silva, E. M., Vieira, C. A., Silva, F. O., \& Ferreira, E. V. (2017). Desafios da enfermagem diante das reações transfusionais, Brasil. Rev. Enferm. UERJ. 25, 11552 . https://www.e-publicacoes.uerj.br/index.php/enfermagemuerj/article/download/11552/22666

Souza V. Segurança e intervenção: a assistência de enfermagem em hemotransfusão. Rio de Janeiro,Tese em Português 2017. https://pesquisa.bvsalud.org.

Souza, M. T., Silva, M. D., \& Carvalho, R., (2010). Revisão integrativa: o que é e como fazer. Einstein. 8(1 Pt 1):102-6 https://www.scielo.br/scielo.php?pid=S1679-45082010000100102\&script=sci_arttext\&tlng=pt

Tavares, J. L, Barrichello, E., Mattia, A. L.de, \& Barbosa, M. H. (2015) Fatores associados ao conhecimento da equipe de enfermagem de um hospital de ensino sobre hemotransfusão. Revista Latino-Americana de Enfermagem. 23(4), 595-602. https://www.redalyc.org/pdf/2814/281442224005.pdf 
Research, Society and Development, v. 10, n. 8, e15310815471, 2021

(CC BY 4.0) | ISSN 2525-3409 | DOI: http://dx.doi.org/10.33448/rsd-v10i8.15471

Teixeira, E., Horácio Pires Medeiros, H. P., Nascimento, M. H. M., Silva, B. A. C., \& Rodrigues, C., (2013). Revisão Integrativa da Literatura passo-a-passo \& convergências com outros métodos de revisão. Rev Enferm UFPI, 2(spe):3-7, https://ojs.ufpi.br/index.php/reufpi/article/view/1457.

Ursi, E. S., \& Galvão, C. M. (2006). Prevenção de lesões de pele no perioperatório: revisão integrativa da literatura. Rev.Latino-am Enfermagem 14(1):124-31. https://www.scielo.br/scielo.php?pid=S0104-11692006000100017\&script=sci_arttext

Vieira, C. M. A. S., \& Santos, K. B. (2020). O conhecimento da equipe de enfermagem em transfusão de hemocomponentes: uma revisão integrativa. Rev Fun Care 12:517-524. http://dx.doi.org/0.9789/2175- 5361.rpcfo.v12.8623. 